\title{
The New Incinerator in Parma and the News from Newspapers - The Importance of Communication in Terms of "Environment and Health"
}

\author{
Alessandra Rampini, ${ }^{1, *}$ Elisa Mariani ${ }^{2}$, Rosanna Giordano ${ }^{2}$, Licia Veronesi ${ }^{3}$, Matteo Goldoni ${ }^{4}$, \\ Giuliano Ezio Sansebastiano ${ }^{3}$, Maurizio Impallomeni \\ ${ }^{1}$ Department of Public Health, Azienda USL di Piacenza, Piazzale Milano 2, 29121 Piacenza, Italy \\ ${ }^{2}$ Department of Public Health, Azienda USL di Parma, Via Vasari 13/A, 43126 Parma, Italy \\ ${ }^{3}$ Department of Medicine and Surgery, University of Parma, Via Volturno 39, 43125 Parma, Italy \\ ${ }^{4}$ Department of Medicine and Surgery, University of Parma, Via Gramsci 14, 43126 Parma, Italy
}

Copyright $@ 2017$ by authors, all rights reserved. Authors agree that this article remains permanently open access under the terms of the Creative Commons Attribution License 4.0 International License

\begin{abstract}
Objective is the evaluating of news concerning the incinerator in Parma and assessing any potential information gap, which could be addressed by institutional communication. Articles from both online and printed newspapers were selected, then underwent to double-blind review and were further evaluated also by a non-partisan expert. 2,390 articles were found through 'Google News' (incinerator, waste-to-energy plant, Parma); 96 of these were selected according to the relevance of the theme, then they were categorized as 'informative', 'scientific', 'political', 'socio-political' and 'economic' by all three evaluators. Such classification was finally contextualized depending on the type of newspaper. The agreement was considered using Cohen's kappa statistic. Within the 96 articles, 3 were economic, 34 political, 29 socio-political, 28 informational and only 2 scientific. The articles were further categorized by type of newspaper: $1 \%$ economic, $1 \%$ political, $5.2 \%$ environmentalist, $15.6 \%$ self-defined independent, 35.4\% national and $41.7 \%$ local newspapers. This review shows that both local and national press are interested in the topic at issue, nevertheless it reveals a lack of scientific information. This analysis has not found articles in which the institutions directly involved have been consulted. Thus it would be desirable to have a greater mutual cooperation between the press and such institutions.
\end{abstract}

Keywords Incinerator, Risk Perception, Communication

\section{Introduction}

Environmental issues are often considered as a central theme in the broad debate on the determinants of health/disease that involves, on one hand the general public and on the other, various stakeholders and policy makers. The assessment and management of environmental risk, in addition to national and supranational regulatory constraints, depends on important territorial aspects that require proper communication, in order to adopt strategies and behaviors shared by both governments and people. The same policies are in turn influenced not only by scientific knowledge and technical available solutions, but also by beliefs, expectations and fears of population.

With regard to the great environmental impact, in fact, the risk perception can be distorted from the actual risk, as it is susceptible to various factors, both individual and social, such as age, sex, socio-cultural status, and proximity to the supposed danger. [1, 2] Moreover, the perceptions and social representations of risks are historically dependent: any period can be characterized by the inputs that identify specific dangers, thus exasperating fears. [3]

The media have a key role in characterizing the attitude towards a specific risk. One of the effects that have been studied in literature concerns the so called "social risk amplification." [4-6] This is a well-known effect of mass media, which tend to increase the importance of certain topics and spread more negative news than positive ones. The attitude of creating a sort of "echo effect" of negative opinions and aspects of the controversial issues is particularly relevant on the internet that gives no room to all parties' opinion and can generate resentment and disaffection towards the institutions.

A recent survey (Eurobarometer 82.3) shows that more than half of the Italians consider the Internet as a credible source of information. Despite this study, most social scientists believe that the press provides a valuable support to democracy by giving visibility to the "impeached party". [7] Journalism in fact, should guarantee a space for the 
diffusion of counter-arguments, which in turn can facilitate the engagement of general public and provide a support to the institutions.

However, through the so-called "agenda setting" methodology even the professionals of press include into the agenda "a set of topics of a certain priority at any given time", according to a specific definition: not all facts become news and not all news becomes great stories. [8] The selection is based on the "newsworthiness", which depends on various factors, including socio-cultural ones (current affairs, proximity, expectations, violation of common norms), narrative (identifiable protagonists, guilty, potential conflicts) and technical. [3] Therefore, it is clear that the presentation of news in the newspapers have often ways that have little to do with the amount of risk. [9]

The agenda is structured according to the relevance that topics have for citizens; the media, actually, allow the general public to decide the agenda by focusing more on certain events and topics and leaving out others. So, according to an organizational approach, the media can be considered a self-centered and autonomous entity. However, in relation to a relational approach, the production of information can be seen as a result of negotiations between the media and all the influences of even opposing forces acting on them. [10]

This process is completed by the "agenda building", according to which the creation of the agenda takes place through the action of a plurality of actors, often outside the context of the media. The "agenda building" changes the content and the outcomes according to the level of knowledge on which the influence of the media acts. The action of "agenda setting" is divided into two aspects, the selection and the prioritization of the issues. People tend to include or exclude information from their own knowledge and give more importance to the topics covered by the media. [11, 12]

The influence of the media does not focus only on the importance of an issue, but it has "the ability to suggest criteria for judgment, or rather, to trigger certain types of judgment through topics" [13]. This mechanism is called "priming” as the topics serve as a trigger to judgment.

The media, subsequently, also create an interpretive framework, in order to give a reading key: they build a framework around the news through a process called "framing", defined as "the selection of some aspects of a perceived reality to make them more salient in a text communication, in order to promote a particular problem definition, causal interpretation, a moral evaluation and/or a suggestion on how to address the issue described". [14] The same topic can be presented differently depending on different cultural background, beliefs, political ideas of the journalist, party interests, social involvement.

The whole process starts by the collection of information from different sources, which are represented by various press offices, agencies but also by population surveys which have been carried out ad hoc, to select some hot topics.

"Environment and health" is definitely a topic that is included both in political (press offices) and social agendas (opinions of key stakeholders) and has elements of conflict - therefore it is of particular interest for the media. In particular, the issue of waste management and, more specifically, incinerators, can be considered as a paradigmatic example of the different aspects that could characterize the communication of environmental risk and health-related issues. [15-17]

More precisely, the goal of this paper is to evaluate the different communication approaches regarding the new incinerator in Parma (Italy), in order to understand quantity and quality of the scientific information that have been reported by newspapers to the population. This exploratory study is conducted by professionals involved in the "Project on the Direct and Indirect Effects of the new Incinerator on Residents in Parma" and the aim is to evaluate a possible informational gap to be filled by appropriate institutional communication interventions. This approach, based on the principles of the Consensus Communication, aims also to understand if the population is aware of the information collected through the surveillance project, which is active since 2011, to promote a comparison between different visions of the problem, and then provide information to the population with adequate means to guarantee a correct risk perception. [2]

\section{Methods}

Articles about the new incinerator in Parma were found through "Google News" web search engine; the following keywords were used: incinerator, waste-to-energy plant and Parma. From the date of February 1st, 2016 and going back to August 11th, 2010, until the last article on, 2390 articles were selected. Title and abstract of every article were read, then each one was selected/excluded using inclusion/exclusion criteria shared a priori.

- $\quad$ Exclusion criteria: blogs or social media comments; articles only from online newspapers.

- Inclusion criteria: articles from both online and printed newspapers, articles concerning the incinerating plant in Parma, for various reasons.

106 articles were hence collected. This first screening was conducted by two double-blind examiners.

Then articles were classified by reading the full text. In order to an interpretive framework, articles were classified in 5 pre-established topic areas as follows:

- Political: articles that address the issue as a mere political propaganda pretext, without any scientific or technical reference

- $\quad$ Socio-political: articles regarding only decisional and social aspects of the affair

- $\quad$ Economic: that focus on economic interests related to the starting of the incinerator and its repercussions on the population's bill 
- Informative: articles appearing as opinions based on any evidence or expertise

- Scientific: articles that report the news based on scientific knowledge and expert advice.

A series of 8 questions has been developed to guess the framework around the news. Each question was meant to measure a specific aspect, identifying information about content, source and tone of the news. Questions were for example: "Does the article report controversy and reflect disagreement between parties?" (framework of conflict) or "Are population benefits and / or disadvantages analyzed?" (framework of human interest). The coders had to answer yes (1) or no (0) for each question. The articles have been classified in a specific topic area if the highest score were corresponding to the sum of the same topic frameworks (table 1). [18-20]

The classification of articles in topic areas was carried out by three evaluators: two inexperienced young (classifiers A and B) and one professor with experience in the field (classifier C).

Then we tried to evaluate the general agreement between the classifications in the 5 categories (political, socio-political, economic, informative, scientific) for the three different evaluators. [21]

Cohen's kappa coefficient was assessed, as follows:
- $\quad$ per subject, using Cohen's kappa with Landis-Koch extension (Response Kappa if $\mathrm{z}($ for $\mathrm{k}=0$ ) Probability)

- $\quad$ per evaluator, employing Crosstabs (Aclassifier vs. B classifier; A classifier vs. C classifier and B classifier vs. C classifier).

According the three evaluators, 10 articles were thus removed because they were considered irrelevant to the topic, resulting in a total number of 96 for the final analysis (figure 1).

In addition, each news organization has been cataloged based on self-definition, according to their school of thought; the following categories have been identified: national, local and economic newspapers, political party magazines, independent and environmentalist newspapers. It was also considered the origin from the different types of newspaper for each of the 96 articles (table 2).

It has also been assessed whether there was a difference of raters' agreement in distribution of articles just for political, socio-political and informative categories (the other ones have too low frequencies), in types of newspapers independent, local and national. We used Fisher-Freeman-Halton exact test, equivalent to the Fisher test with a $3 * 3$ crosstab.

Table 1. Framing questions and relative topic areas

\begin{tabular}{|c|c|c|c|c|c|}
\hline Framing questions & Political & Socio-political & Economic & Informative & Scientific \\
\hline Does the article report controversy? Is the style impartial? & & & & Yes (1) & \\
\hline Does the article reflect disagreement between parties? & Yes (1) & Yes (1) & & & \\
\hline Are statements evidence-based (facts / interviews)? & & & Yes (1) & Yes (1) & Yes (1) \\
\hline Are there economic and financial statements? & & & Yes (1) & & \\
\hline Are population benefits and / or disadvantages analyzed? & & Yes (1) & & Yes (1) & \\
\hline $\begin{array}{l}\text { Is there un attribution of guilty / responsibility to governors or } \\
\text { parties? }\end{array}$ & Yes (1) & & & & \\
\hline Are scientific sources and/or expert opinions mentioned? & & & & & Yes (1) \\
\hline Is the style persuasive, drawn and flowery? & Yes (1) & Yes (1) & & & \\
\hline
\end{tabular}

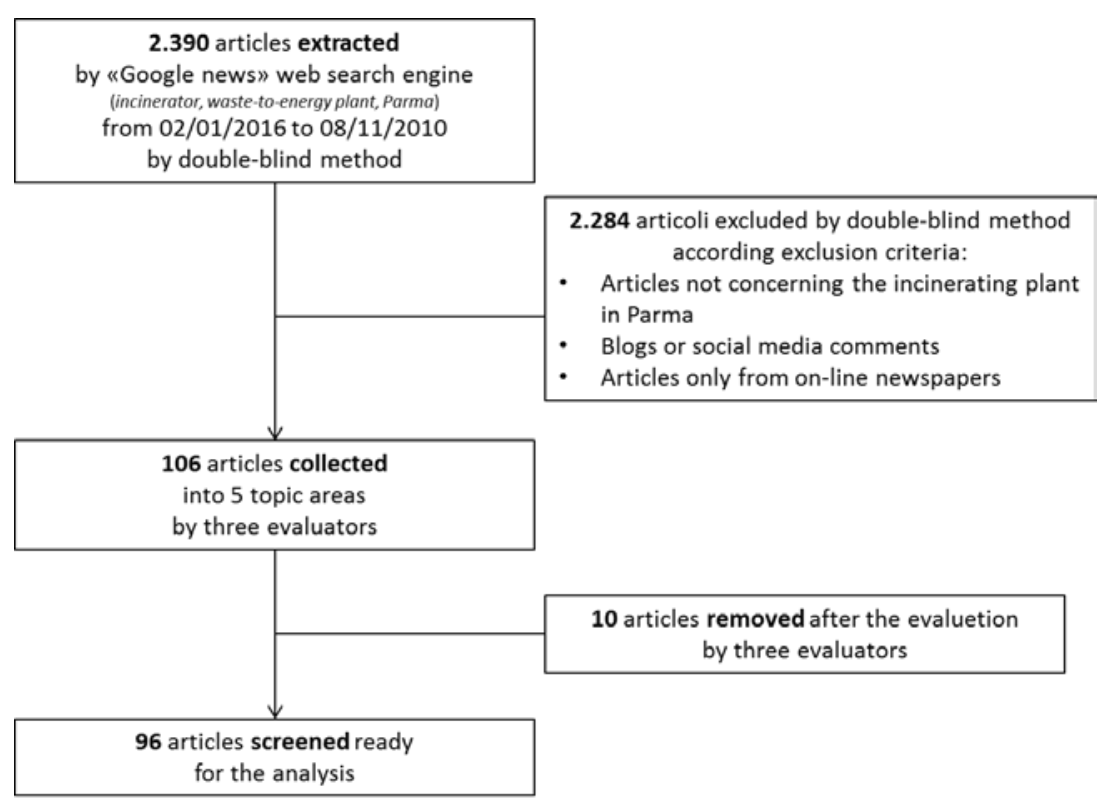

Figure 1. Flow chart of the various steps that led to the selection of 96 newspaper articles considered 
Table 2. Matrix used for the review in double and triple blind, depending on the type of newspaper and thematic areas in which the articles were classified

\begin{tabular}{|c|c|c|c|}
\hline DATE & TITLE & KIND OF NEWS ORGANIZATIONS & TOPIC AREAS \\
\hline $2010-12-06$ & Da Parma a Roma si accende il dibattito sugli inceneritori & environmentalist & informative \\
\hline 2011-02-23 & È incredibile! Pure la rete di teleriscaldamento dell'inceneritore sarebbe illegittima! & local newspaper & socio-political \\
\hline 2011-07-01 & Dal Comune stop all'inceneritore. Iren: "Ci tuteleremo", Gcr festeggia & local newspaper & socio-political \\
\hline 2011-10-24 & Termovalorizzatore Uguzzolo: le rassicurazioni di Barilla & independent & informative \\
\hline 2011-12-19 & Inceneritori, serio rischio per la salute: i risultati del progetto Moniter & environmentalist & scientific \\
\hline $2012-01-20$ & Inceneritore, altro che richiamo dall'Ue. Ealla Certosa un altro forno... & local newspaper & informative \\
\hline 2012-01-24 & Parma, richiamo dell'Europa per l'inceneritore. "Nascosti i conti e violata la concorrenza" & national newspaper & political \\
\hline $2012-05-17$ & Parma, mille persone al confronto sull'inceneritore: la vittoria passa da qui & national newspaper & political \\
\hline $2012-05-21$ & Pizzarotti, sindaco "No termo". II fattore "inceneritore" nella vittoria del Movimento 5 stelle a Parma & local newspaper & political \\
\hline $2012-06-09$ & II commercialista, I'imprenditore e un environmentalist: ecco gli assessori chiave del grilllino Pizzarotti & economics & political \\
\hline 2012-08-22 & Iren, Parma alla guerra dell'inceneritore & national newspaper & political \\
\hline $2012-09-02$ & Inceneritore, Iren rischia 40 milioni di incentivi & local newspaper & political \\
\hline $2012-09-20$ & II mistero dell'inceneritore di Parma & independent & political \\
\hline 2012-12-07 & Le novità sull'inceneritore di Parma & national newspaper & political \\
\hline 2012-12-12 & II Riesame è contro il sequestro dell'inceneritore di Parma (notizia che non leggerete su beppegrillo.it) & national newspaper & political \\
\hline 2013-01-03 & Parma, la giunta dei grillini delude: Imu cara, rette d'asilo sempre più alte e conti in rosso & national newspaper & political \\
\hline 2013-01-03 & Federico Pizzarotti e la delusione grillina a Parma & independent & political \\
\hline 2013-03-02 & L'inceneritore di Parma è stato acceso & national newspaper & informative \\
\hline 2013-03-09 & Tifare Pizzarotti. Di questi tempi & national newspaper & political \\
\hline 2013-03-13 & Parma, parte l'inceneritore. La resa del sindaco 5 stelle & national newspaper & socio-political \\
\hline 2013-03-27 & Parma si arrende all'inceneritore & environmentalist & informative \\
\hline 2013-04-29 & "Il Movimento 5 stelle è una bolla di sapone" & independent & political \\
\hline 2013-04-30 & E a Parma si accende l'inceneritore di Pizzarotti & independent & political \\
\hline 2013-05-02 & Termovalorizzatore di Parma: esistono impianti in grado di tutelare la salute? & environmentalist & informative \\
\hline 2013-05-15 & Più sindaco che grillino: un anno di Pizzarotti & political & political \\
\hline 2013-05-17 & Pizzarotti mette alla porta i manifestanti anti-inceneritore & independent & political \\
\hline 2013-05-30 & Inceneritore di Parma: quello che Pizzarotti non dice & independent & political \\
\hline 2013-06-04 & Inceneritore di Parma: stop agli incentivi verdi & independent & informative \\
\hline $2013-06-28$ & Inceneritore, Allegri e i No Termo all'attacco: "II comune deve cacciare Iren" & local newspaper & political \\
\hline 2013-08-01 & Inceneritore di Uguzzolo, Andrea Viero: "Sarà a regime dal $1^{\circ}$ agosto" & local newspaper & socio-political \\
\hline 2013-08-09 & Anonymous contro l'inceneritore di Parma & independent & socio-political \\
\hline 2013-08-12 & La grande partita dei rifiuti in Emilia Romagna & national newspaper & political \\
\hline $2013-08-28$ & Acceso l'inceneritore di Parma. Pizzarotti: "Ho fatto il possibile per fermarlo" & national newspaper & political \\
\hline 2013-08-30 & Beppe Grillo e il parmigiano alla diossina & independent & socio-political \\
\hline 2013-09-03 & Beppe Grillo e le accuse contro l'inceneritore di Parma & independent & socio-political \\
\hline 2013-09-04 & II consorzio del prosciutto di Parma contro Beppe Grillo & independent & informative \\
\hline 2013-09-04 & Emissioni da inceneritori ridotte da 1000 a 50 volte in 50 anni & environmentalist & scientific \\
\hline 2013-10-04 & L'inceneritore ha già prodotto 5mila tonnellate di ceneri. Gcr: "Ma che fine fanno?" & local newspaper & informative \\
\hline 2013-10-20 & Inceneritore, lavori ancora in corso. Gcr: "Ma il permesso è scaduto da 5 giorni" & local newspaper & socio-political \\
\hline 2013-11-16 & Parma, occupato I'inceneritore di Ugozzolo: manifestanti bloccano camion di rifiuti & national newspaper & socio-political \\
\hline 2013-11-20 & Dentro il termovalorizzatore "Qui le migliori tecnologie" & national newspaper & informative \\
\hline 2013-12-04 & Viaggio nel termovalorizzatore di Parma, il mostro secondo Grillo & national newspaper & informative \\
\hline 2013-12-10 & Inquinamento, il Natale nero di Parma. El'inceneritore non si fermerà & local newspaper & socio-political \\
\hline 2013-12-30 & Rifiuti, sindaci emiliani a Regione e ministro: "Inceneritori, serve un piano" & national newspaper & socio-political \\
\hline 2014-02-01 & Come i politici manipolano i dati sull'inquinamento & local newspaper & socio-political \\
\hline 2014-02-07 & Teleriscaldamento in ritardo di un anno. Iren chiede nuovi permessi alla Provincia & local newspaper & socio-political \\
\hline 2014-02-28 & Luce verde anche all'ultimo pezzo dell'inceneritore & local newspaper & informative \\
\hline 2014-03-03 & Inceneritore di Parma, primo sforamento ai limiti di emissione & local newspaper & informative \\
\hline 2014-04-08 & Grillo sfiducia il sindaco di Parma "Capitan Pizza perché parli?" & national newspaper & political \\
\hline 2014-04-21 & Inceneritore, l'assessore all'Ambiente: "Casaleggio pontifica senza sapere" & national newspaper & political \\
\hline 2014-05-19 & Dopo due anni, Pizzarotti ha mantenuto solo un decimo delle promesse elettorali del 2012 & local newspaper & political \\
\hline 2014-08-01 & Inceneritore, la denuncia del Gcr: "18 incidenti dall'attivazione" & local newspaper & informative \\
\hline
\end{tabular}




\begin{tabular}{|c|c|c|c|}
\hline DATE & TITLE & KIND OF NEWS ORGANIZATIONS & TOPIC AREAS \\
\hline 2014-09-19 & Sull'inceneritore di Parma lo spettro dei rifiuti del sud & national newspaper & socio-political \\
\hline 2014-09-21 & Inceneritore Parma, Tar boccia Iren: "Non può bruciare rifiuti da fuori provincia" & national newspaper & socio-political \\
\hline 2015-01-08 & I rifiuti di Parma i più cari della regione & local newspaper & political \\
\hline 2015-02-18 & Inceneritore, l'Anticorruzione: "Si paghino oneri di urbanizzazione" & national newspaper & informative \\
\hline 2015-03-25 & Inceneritore, le proteste di Via Sagarat e i problemi del teleriscaldamento & local newspaper & socio-political \\
\hline 2015-03-27 & Inceneritore, la rete di teleriscaldamento è allacciata" & local newspaper & informative \\
\hline 2015-04-17 & Inceneritore, Iren controllore e controllato? Arpa: "Nessun conflitto di interessi" & local newspaper & informative \\
\hline 2015-05-09 & Rifiuti, "la raccolta notturna puntuale è piena di difetti" & local newspaper & REMOVED \\
\hline 2015-05-22 & Parma, Iren vuole più rifiuti nell'inceneritore di Uguzzolo & independent & informative \\
\hline 2015-05-22 & Inceneritore, avviato l'iter per bruciare rifiuti da tutta Italia. Iren: "Nessun impatto per l'ambiente" & national newspaper & socio-political \\
\hline 2015-06-12 & Rifiuti da fuori provincia: 11 luglio, manifestazione a Parma & local newspaper & socio-political \\
\hline 2015-06-19 & "Pizzarotti poteva fermare l'inceneritore di Parma" & independent & socio-political \\
\hline 2015-06-20 & Pd: inceneritore, Bagnacani vota "contro" il Comune? & local newspaper & socio-political \\
\hline 2015-06-25 & Legambiente: "L'11 luglio in piazza, ci sia tutta la città" & national newspaper & socio-political \\
\hline 2015-07-10 & Termovalorizzatori, accordo con Hera e Iren per limitare I'utilizzo degli impianti & national newspaper & socio-political \\
\hline 2015-07-10 & Inceneritore, slitta il voto dei sindaci sul potenziamento chiesto da Iren & national newspaper & socio-political \\
\hline 2015-07-14 & Emergenza rifiuti, il pattume della Liguria negli inceneritori emiliani & local newspaper & informative \\
\hline 2015-07-15 & Ipotesi rifiuti in arrivo dalla Liguria & national newspaper & socio-political \\
\hline $2015-07-20$ & Inceneritore, associazioni ambientaliste: "Provincia respinga richiesta di Iren" & national newspaper & |informative \\
\hline 2015-07-22 & Rifiuti dalla Liguria in Emilia, Parma si oppone. La Regione pensa all'inceneritore di Piacenza & national newspaper & socio-political \\
\hline 2015-07-27 & Inceneritore, dall'Assemblea dei Sindaci un convinto no al potenziamento della capacità di smaltimento & local newspaper & socio-political \\
\hline 2015-08-20 & Iren vuole costruire due nuovi inceneritori & local newspaper & informative \\
\hline 2015-08-27 & Iren, sale l'utile. Boom di ricavi per rifiuti & national newspaper & economics \\
\hline 2015-09-02 & Folli e il bando Iren: "Come si spiega il ribasso 20\%?" & local newspaper & REMOVED \\
\hline 2015-09-09 & Sblocca Italia: Renzi accelera sugli inceneritori per placare l'Europa & national newspaper & REMOVED \\
\hline 2015-09-30 & Rifiuti, approvata la nuova legge regionale. Bonaccini: "Spegneremo inceneritori" & national newspaper & REMOVED \\
\hline 2015-09-30 & Rifiuti, approvata la nuova legge regionale. Bonaccini: "Potremmo spegnere inceneritori" & local newspaper & REMOVED \\
\hline 2015-10-08 & Raccolta differenziata, Pizzarotti relatore al convegno di Legambiente a Roma & local newspaper & REMOVED \\
\hline 2015-10-08 & forum rifiuti legambiente: Pizzarotti relatore a Roma & environmentalist & REMOVED \\
\hline 2015-10-19 & "Teleriscaldamento Iren illegittimo: serviva gara d'appalto" & local newspaper & informative \\
\hline 2015-11-12 & Iren: boom di ricavi dai rifiuti. Che bruciano a Parma & local newspaper & economics \\
\hline 2015-11-16 & Consiglio provinciale, avanti col monitoraggio degli effetti dell'inceneritore & national newspaper & informative \\
\hline 2015-11-24 & Rifiuti, Parma prima in Emilia per costo della raccolta & national newspaper & economics \\
\hline 2015-11-30 & Pellacini: "Rifiuti a Parma come a Livorno e intanto I'inceneritore brucia” & local newspaper & socio-political \\
\hline 2015-12-09 & Come governano i grillini al potere & national newspaper & REMOVED \\
\hline 2015-12-10 & Pizzarotti rilancia:"Incontro aperto a tutti gli eletti" e torna ad invitare Di Maio a Parma & national newspaper & political \\
\hline 2015-12-14 & Inceneritore, incarico per l'analisi delle emissioni & local newspaper & informative \\
\hline 2015-12-14 & Inceneritore: il Comune cerca un tecnico per le analisi sulle emissioni & local newspaper & linformative \\
\hline 2015-12-14 & Inceneritore, Comune: incarico professionale perl'analisi delle emissioni & local newspaper & informative \\
\hline 2015-12-26 & Gcr e inceneritore: scintille fra Dall'Olio e Folli & local newspaper & political \\
\hline 2015-12-27 & Folli, il GCR e l'Arpa. Dall'Olio (PD): “Tutti i regali di Natale al GCR”. Replica e controreplica & local newspaper & political \\
\hline 2016-01-03 & A Parma andrà I'80 per cento dei rifiuti "reggiani" & local newspaper & informative \\
\hline 2016-01-04 & I rifiuti di Reggio Emilia arriveranno a Parma? & national newspaper & political \\
\hline 2016-01-04 & I soldi di Iren non puzzano nemmeno per Folli & local newspaper & political \\
\hline 2016-01-08 & Piano regionale rifiuti, la soddisfazione del Pd di Parma & national newspaper & socio-political \\
\hline 2016-01-08 & Scambio di rifiuti con Parma, Piacenza dice no. "Più costi e salute a rischio" & local newspaper & political \\
\hline 2016-01-08 & A Parma i rifiuti di Reggio. II Pd: "Tariffe più basse del 10\%" & local newspaper & political \\
\hline 2016-01-08 & Rifiuti, Regione vara accordo per Forli. Parma resta in attesa & national newspaper & REMOVED \\
\hline 2016-01-08 & I rifiuti di Reggio finiranno nell'inceneritore di Parma & independent & informative \\
\hline 2016-01-09 & Rifiuti da fuori nell' inceneritore di Parma, bocciata la politica di Pizzarotti. Le accuse di Pellacini e Ghiretti & local newspaper & political \\
\hline 2016-01-10 & Rifiuti da Reggio, il Pd: "II tradimento è dei 5 stelle" & local newspaper & political \\
\hline 2016-01-11 & Nuovi inceneritori: le ragioni del no & environmentalist & REMOVED \\
\hline 2016-01-12 & Inceneritore, PD: "II doppio gioco di Pizzarotti" & local newspaper & political \\
\hline 2016-01-12 & Inceneritore di Parma, scambio di accuse Pd- Pizzarotti & national newspaper & socio-political \\
\hline
\end{tabular}


A further analysis was conducted considering the dates of publication in order to assess the possible influence of social events or interesting situations on interpretive frameworks.

\section{Results}

Of the 96 articles reviewed, 3 were classified as economic, 34 as political, 29 as socio-political, 28 as informative and only two as scientific. None of this treated of the "Project on the Direct and Indirect Effects of the new Incinerator on Residents in Parma".

These data were obtained considering the classification of the three evaluators and their agreement. The latter can be defined as good as the outcome of the statistical tests below (table 3).

It concludes that agreement is excellent between observers $\mathrm{A}$ and $\mathrm{C}$, good between observers $\mathrm{A}$ and $\mathrm{B}$ and good also between observers B and C. Note that there is agreement between both young evaluators as well as between each young observer and the experienced one (table 4). Besides considering the type of organization behind the news, according to self-definition of each newspaper, the articles were categorized as: $1 \%$ economic, $1 \%$ political, $5.2 \%$ environmentalist, $15.6 \%$ self-defined independent newspapers, 35.4\% national and $41.7 \%$ local newspapers (figure 2).

Table 3. General agreement over all (political, socio-political, economic, informative, scientific) categories with 3 raters per subject

\begin{tabular}{|c|c|}
\hline \multicolumn{2}{|c|}{ General agreement over all (5) categories with 3 raters per subject } \\
\hline Cohen's kappa & Universal (Berry-Mielke) $\mathrm{R}$ \\
\hline (Landis-Koch extension) & \\
Response Kappa if $\mathrm{z}$ (for $\mathrm{k}=0$ ) & \\
Probability Combined & Weighted (linear) kappa $=$ \\
(Fleiss-Nee-Landis test) & 0.553073 \\
Kappa* $=0.757759$ & $\mathrm{P}<0.0001$ \\
95\% CI: 0.684597 to 0.830921 & \\
$\mathrm{z}$ (for $\mathrm{k}=0$ ) $=20.299866$ & \\
$\mathrm{P}<0.0001$ & \\
\hline
\end{tabular}

*Note that there is good concordance if $\mathrm{k}$ takes values between 0.6-0.8

Table 4. General agreement over all (political, socio-political, economic, informative, scientific) categories with 2 raters per subject

\begin{tabular}{|c|c|c|c|}
\hline \multicolumn{4}{|c|}{ General agreement over all (5) categories with 2 raters per subject } \\
\hline Cohen's kappa (unweighted) & A vs. B & A vs. C & B vs. C \\
\hline Observed agreement & $81.25 \%$ & $91.67 \%$ & $76.04 \%$ \\
\hline Expected agreement & $29.49 \%$ & $30.27 \%$ & $29.25 \%$ \\
\hline Kappa* & $\begin{array}{c}0.734072 \\
\left(\mathrm{se} \_0=0.064377, \mathrm{se}=\right. \\
0.056523)\end{array}$ & $\begin{array}{c}0.880486 \\
(\text { se_0 }=0.065103, \text { se }=0.04045)\end{array}$ & $\begin{array}{c}0.66135 \\
\left(\mathrm{se} \_0=0.063534, \mathrm{se}=\right. \\
0.061268)\end{array}$ \\
\hline 95\% confidence interval & 0.623288 to 0.844856 & 0.801206 to 0.959766 & 0.541266 to 0.781433 \\
\hline $\mathrm{z}($ for $\mathrm{k}=0$ ) & 11.4027619331458 & 13.5244969257769 & 10.4093671906293 \\
\hline $\mathrm{P}$ & $<0.0001$ & $<0.0001$ & $<0.0001$ \\
\hline
\end{tabular}

*Note that there is good concordance if $\mathrm{k}$ takes values between $0.6-0.8$

\section{A. Classification of the articles according to the thematic areas}

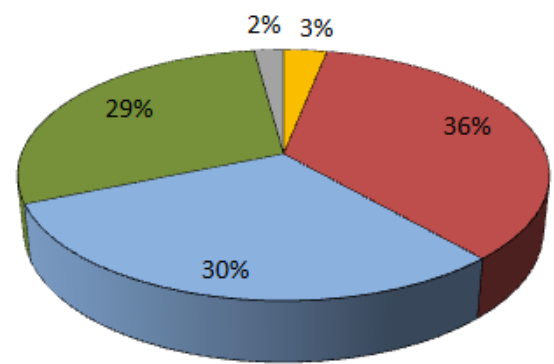

\section{B. Distribution of the articles into different} type of newspaper

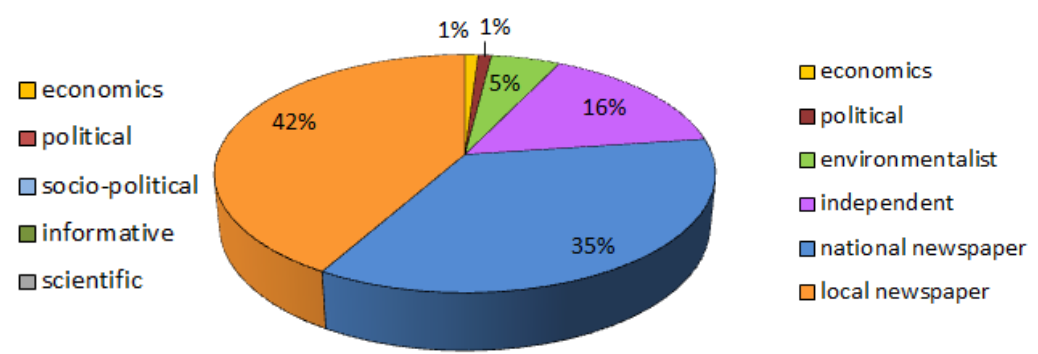

Figure 2. Classification of the articles selected according to the thematic areas (A) and in relation to the type of newspaper (B) 


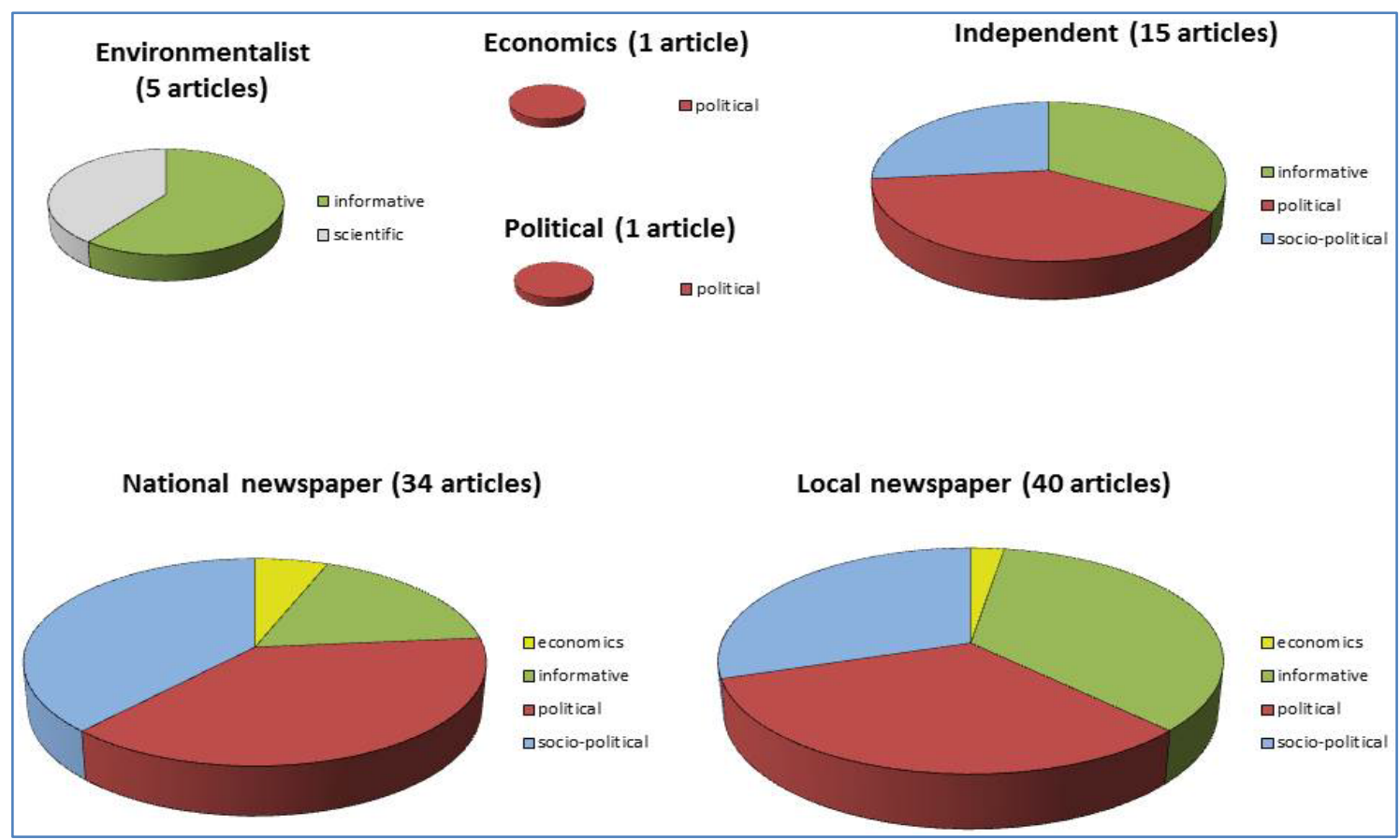

Figure 3. Classification of articles by topic according to their distribution in various newspapers

By integrating these results one another, it was found that (figure 3):

- The self-defined environmental magazines (5 items in total) have provided informative (60\%) and scientific (40\%) articles

- $\quad$ Both economic and political newspapers (one item each) reported only articles of a political nature

- Independent newspapers (15 items in total) have focused mostly on political issues (40\%), however reporting also informative (33.3\%) and socio-political (26.7\%) ones

- $\quad$ Even national newspapers (34 items in total) have treated the subject, especially in political (38.2\%) and socio-political (38.2\%) terms, spending less attention to the informational (17.6\%) and economical (5.9\%) aspects

- Finally, local newspapers have devoted many articles (40 items in total) to the matter in hand, describing it in equal measure through political (32.5\%), socio-political (30\%) and informative (35\%) aspects, while only briefly citing the economics ones $(2.5 \%)$.

As for the correlation in classifiers, possible differences in agreement between the classification of the most frequent articles (political, socio-political and informative) have been evaluated for three types of newspapers (independent, local, national). The result is that the distribution is about the same (Fisher-Freeman-Halton exact $\mathrm{P}=0.6226$ ).

Then the articles were evaluated according to their publication date and they were related to events of particular interest. It emerged that the informative and socio-political articles are distributed fairly steadily along the time axis considered. Conversely, the only two scientific papers date back to the period immediately following the construction of the new incinerator and the start phase of the incinerator activity.

The economics articles have been published all in conjunction with the decision to implement combustion by receiving municipal solid waste also from areas nearby the province of Parma, highlighting its impact on operating costs and the possible change in bill rates. As for the articles that deal with the issue for political purposes, these ones are distributed in three temporal clusters that coincide with the following events: peri-election period (2012 municipal elections), the days close to the start of full incinerator activities and immediately after the confirmation of the arrival in Parma of municipal solid waste from Reggio Emilia (figure 4). 


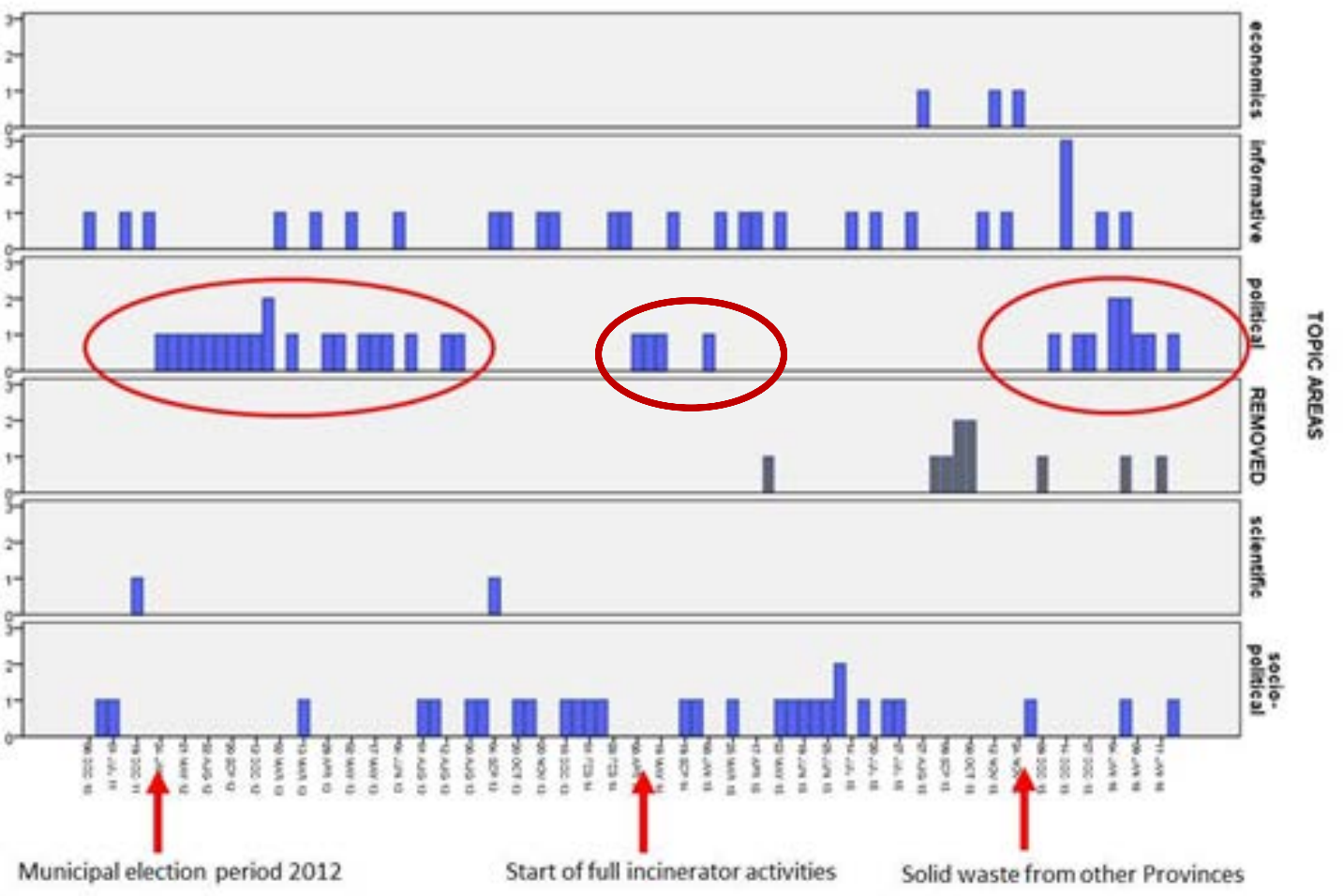

Figure 4. Timing of the various items according to their classification and the dates of publication put in relation with some of the events considered to be of particular interest

\section{Discussion}

As suggest on CDC's Guidelines for Evaluating Public Health Surveillance Systems, the evaluation of a public health surveillance project should also valuate communication skills and, in particular, to assess how well the surveillance system is integrated with other information systems. [22, 23]

So, some professionals involved in the "Project on the Direct and Indirect Effects of the new Incinerator on Residents in Parma" tried to collected news concerning the affair of incinerator in Parma to understand quantity and quality of the scientific information that have been reported by newspapers to the population.

We analyzed only articles from the newspapers because they are considered more professional information sources among the media. The decision to consider just the newspapers, both in print and online, depended on the intention to analyze information able to reach a wide and heterogeneous population.

We are aware that the theme "environment and health" is open to the development of personal opinions; however, we tried to make the analysis as much objective as possible, following a survey methodology similar to the one of systematic reviews, classifying both newspapers and individual articles. The review of the articles was not only quantitative but also qualitative, evaluating the different interpretive frameworks of discussion of the story. Then the consistency between the classifications of the three different evaluators and the disagreement between items were considered using Cohen's k. Cohen's kappa coefficient is a statistic which measures inter-rater agreement for qualitative items. It is a more robust measure than simple percent agreement calculation, since $k$ takes into account the possibility of the agreement occurring by chance. [19] We have considered the role of expert/inexperienced coders: the agreement was good both among young reviewers, and between the experienced professor and each young classifier. In this way, we have tried to analyze with a science-based approach a world that usually lends to opinions, such as news.

This gamble has been necessary to understand the kind of news that has reached most of the population resident in Parma and to highlight any information gap.

Most of the items we considered were so classified as political (35.4\%) and socio-political (30.2\%), which demonstrates how the interest to incinerators is involving the political world in all its aspects. In particular, it has been observed that in Parma, the coincidence between the upcoming municipal elections and the realization of the new incinerator has favored the use of the issue for propaganda purposes. We underline that the content of most of the articles focuses precisely on the political struggle against the new incinerator in progress and on the promise of the future mayor of preventing it from completing. Once the incineration plant was completed, then started and later its activity boosted, the media battle was increasingly fired against the municipal administration that failed to do so they promised (as you can see from the publication time-line). This fact probably means that the 
press has focused mainly on political matters at the expense of a more objective and scientific information.

In this situation, the new incinerator was seen as a dangerous plant that should never have been realized. It reaffirms that in political and socio-political articles no technical-scientific data has been reported to support this topic. It is hence reasonable to think that the majority of the population received distorted information that is limited only to certain aspects.

While analyzing the types of newspapers that have dealt incinerator in Parma, it is interesting to notice that the story has not only affected the local news ( $41.7 \%$ of articles read) but also the national ones (35.4\%). It should also be noticed that the national press has focused more on the political and socio-political implications (76.4\%) compared to the local press (62.5\%).

Furthermore, unlike what we might have expected, the environmental magazines have written just a few articles (5 in total) on the subject. It should however be emphasized that these magazines have been the only ones to provide rather impartial information (3 informative and 2 scientific articles), in contrast to self-defined independent newspapers, which in $40 \%$ of cases have published articles in which the incinerator appears as a pretext for political propaganda.

So, over the nearly 6 years in question, the population has been "exposed" only on 2 occasions (2.1\%) to newspaper articles that would take into account technical and scientific aspects. Over more, there were no references to the local surveillance project in these articles, but only technical-scientific news dealing with incinerators and health in a more general context.

It should be emphasized that both ARPAE and the Department of Public Health, the scientific institutions involved in the project of monitoring the effects of the incinerator on the health of residents in Parma, have long attempted to meet this need by collecting, analyzing and reporting data on the surveillance in objective and scientific way, as you can see by visiting their corporate sites

(www.ausl.pr.it/azienda/sorveglianza_inceneritore_parma sorveglianza_inceneritore_par/default.aspx;

www.arpae.it/dettaglio_generale.asp?id=2648\&idlivello= 1798).

It is evident that this method of communication cannot be considered sufficient to reach a large proportion of population. However, it was assumed that journalists (especially those of major newspapers) would gather information also from these official sources. We were surprised to note from the collected data that this did not happen.

This attitude could be explained by the fact that the scientific argumentation is often considered, both from reporters and population, a topic for just a few people because of difficult understanding. We believe, instead, that the correct scientific information is intended to make concepts not usually treated understandable.
We therefore believe that it is important for institutions to perceive the quantity and quality of information reaching the population, to guide and improve communication tools, involve media and stakeholders. Data obtained from this review increase awareness of need of engage also other communication channels from institutions involved in the surveillance project. In fact, we are aware that good communication consists in the ability to spread the scientific contents, using clear language and effective tools. The responsibility of communicators is characterized by the appropriate use of the various communication tools, disseminating truthful and understandable information, with the ultimate aim to involve and empower the entire community. [24]

\section{Conclusions}

This analysis is not intended to interfere with freedom of the press, fundamental basis of a free and democratic communication, but to test the perception of risk by the population through information received from newspapers. In this regard, there is an important scientific information gap that could be filled by institutional communication also by press. In fact, in the material analyzed, do not appear articles in which the point of view of the institutions directly involved in the environmental and health surveillance is expressed.

It should be stressed how the institutions have always been committed to proper communication to the population, by formal pathways, as well as their corporate sites. But this information has not reach the population by press.

A good public health surveillance project should be more integrated with other informative systems to think of common general strategies and to promote health literacy in populations. [25, 26]

So it would be desirable to have a greater mutual cooperation between the press and the institutions in order to provide all the available information and make it more affordable, so that everyone can create an opinion as objective as possible.

\section{Conflicts of Interest Declared}

None.

\section{Acknowledgements}

Thanks to Elisa Landini and Laura Faccini.

\section{REFERENCES}

[1] Flynn J, Slovic P, Mertz C, Gender K. Race and perception of environmental health risk. Risk Anal 1994; 14(6): 
1101-1108.

[2] Albanesi C, Pietrantoni L, Zani B et Al. La comunicazione istituzionale dei rischi. Linee guida ministero della Salute 2011.

[3] Sturloni G. Interagire con i mass media. Master in Comunicazione della Scienza, SISSA 2010.

[4] Kasperson R, Renn O, Slovic P, Brown H, Emel J, Goble R, Kasperson J, Ratick S. The Social Amplification of Risk: A Conceptual Framework. Risk Anal 1988; 8: 177-187.

[5] Fischoff B, Slovic P, Lichtenstein S. The public vs the experts. Advances in risk Analysis 1980; 235-249.

[6] Slovic P, Fischhoff B, Lichetenstein S. Characterizing Perceived Risk, in Kates R. W, Hohenemser C, Kasperson J. X. (eds.) Perilous Progress: Managing the Hazards of Technology. Westview Press, Boulder, CO 1985.

[7] Cameron A. Journal of Computer-Mediated Communication Internet, News, and Political Trust: the Difference between Social Media and Online Media Outlets, 2017.

[8] Dearing JW, Rogers EM. Agenda-setting. Thousand Oaks 1996; CA: Sage.

[9] Benton M, Frazier J. The agenda setting function of the mass media at three levels of information holding. Communication Research 1976; 3: 261-74.

[10] Mancini P. Come vincere le elezioni. Il Mulino 1988.

[11] McCombs ME, Donald LS. Function of Mass Media. The Public Opinion Quarterly 1972; Vol. 36 (2): 176-187.

[12] Shaw EF. Agenda Setting and Mass Communication Theory. Gazette, International Journal for Mass Comunication Studies 1979; Vol. XXV (2).

[13] Marini R. Mass media e discussione pubblica: Le teorie dell'agenda Setting. Libri del Tempo, Editori Laterza 2006.

[14] McCombs ME. I media e le nostre rappresentazioni della realtà. Un'analisi della seconda dimensione dell'agenda setting. Bentivegna 1996.

[15] Apruzzese F0S. La percezione del rischio: Metodologia e casi di studio. Quaderni di Moniter 2011; 05.
[16] Cancila E, Ottolenghi M, Tollar F. Inceneritori e conflitti ambientali. Dinamiche ed esperienze di comunicazione e coinvolgimento. Quaderni di Moniter 2009; 01.

[17] Sampaolo L, Tommaso G et Al. The perception of the relationship between environment and health according to data from Italian Behavioural Risk Factor Surveillance System (PASSI). Epidemiol Prev 2017; 41 (1): 20-28DOI: 10.19191/EP17.1.P020.008

[18] Semetko H. A, Valkenburg P. M. Framing European Politics: A Content Analysis of Press and Television News. Journal of Communication, June 2000.

[19] Bubela T. M, Caulfield T. A. Do the print media "hype" genetic research? A comparison of newspaper stories and peer-reviewed research papers. Canadian Medical Association J, Apr. 27, 2004; 170 (9).

[20] Ramdass D, Seshasai S. Document Classification for Newspaper Articles. Document classification for newspaper articles, 2009.

[21] Carletta J. Squibs and Discussions Assessing Agreement on Classification Tasks: The Kappa Statistic. Computational Linguistics, 1996/ Volume 22, Number 2.

[22] CDC. Updated Guidelines for Evaluating Public Health Surveillance Systems, MMWR Recommendations and Reports, July 27, 2001 / 50(RR13); 1-35.

[23] Pontius R, Millones M. Death to Kappa: birth of quantity disagreement and allocation disagreement for accuracy assessment. International Journal of Remote Sensing. 2011; 32: 4407-4429.

[24] Angela P. Verso la strategia nazionale per la biodiversità. Esiti del tavolo tecnico. Il ruolo dell' informazione e della comunicazione come fattori di facilitazione nei processi di condivisione delle strategie decisionali. Ministero dell'ambiente e della tutela del territorio e del mare 2010.

[25] Nutbeam D, The evolving concept of health literacy. Social Science \& Medicine. Volume 67, Issue 12, 2008/12; 2072-2078.

[26] Finn S, O’Fallon L. The Emergence of Environmental Health Literacy-From Its Roots to Its Future Potential. Environ Health Perspect; April/2017. DOI:10.1289/ehp.1409337. 